\title{
Magnetic Reconnection in the Solar Corona
}

\author{
Mukul Kumar and Chi Wang \\ State Key Laboratory of Space Weather, National Space Science Center, \\ Chinese Academy of Sciences, Beijing, China-100190
}

E mail (mukul.nssc@gmail.com).

Accepted: 19 July 2019

\begin{abstract}
Magnetic reconnection, a ubiquitous process in space plasmas, is currently cutting-edge research. This process is usually referred to as the topological reconstruction of the magnetic field in plasmas. Magnetic reconnection in the solar corona has become widely accepted as a key mechanism responsible for the occurrence of solar eruptions such as solar flares and coronal mass ejections. The magnetic energy released in this process is further converted to thermal and kinetic energy which lead to an acceleration of the particles. Hence, research in magnetic reconnection is crucial to better understand the physical mechanisms that trigger solar eruptive events. The present review article particularly focuses on the steady-state MHD theory of slow as well as fast magnetic reconnection and presents observational evidence for magnetic reconnection found in space-based data.
\end{abstract}

(c) 2019 BBSCS RN SWS. All rights reserved

Keywords: Magnetic Reconnection, Solar Corona

\section{Introduction:}

Considered as a global phenomenon, magnetic reconnection is the re-arrangement of the magnetic field topology, which leads to such violent phenomena such as solar flares, Coronal Mass Ejections (CMEs), sawtooth relaxation, tearing instabilities in magnetic confinement fusion etc. (Biskamp 2000, Zweibel and Yamada 2009, Yamada et al. 2010). The theory of magnetic reconnection was historically proposed by (Giovanelli 1946) for explaining the fast energy release observed in solar flares and was further applied to Earth's magnetosphere by Dungey in 1953, who suggested that "The lines of force can be broken and rejoined" (Dungey 1953). This was followed by Sweet who proposed a model suggesting that the union of two bipolar regions at an $\mathrm{X}$-type neutral point flattened the magnetic field to form a current sheet (Sweet 1958). It was Parker (1957) who invented the expression "reconnection of field lines". In addition, Sweet (1958) and Parker (1957), were the first to tackle magnetic reconnection as a local problem considering the plasma inflow and outflow connected through the diffusion region.

Magnetic reconnection has been widely studied in the magnetospheres of planets (e.g. Kivelson and Russell 1995; Treumann and Baumjohann 1997; Scholer 2003) and in laboratory plasmas and tokamak plasmas (e.g., Bellan 2002). Observations are needed to test theory/ models, and in recent years evidence for magnetic reconnection has been searched for in data. Hence, this review article focuses both on theoretical as well as observational developments in magnetic reconnection. In nature, there exist many transient phenomena (e.g., solar flares, magnetospheric substorms, and $\gamma$-ray bursts), which are associated with magnetic activity. It is believed that magnetic reconnection is the fundamental process responsible for these transient phenomena e.g., for solar flares (Shibata 1996a), for occurrence of CMEs ( Lin and Forbes 2000), for the eruptions of filament (Chen and Shibata 2000, Shen et al. 2012, Zhou et al. 2017), for jets (Shibata et al. 1996b, Jiang et al. 2013), for explosive events (Innes et al. 1997), and for coronal bright points (Priest et al. 1994).

The growing interest in the physics of magnetic reconnection has inspired experimental studies in the laboratories. The utilization of computer simulations as a research tool for understanding magnetic reconnection has particularly accelerated, specifically performing numerical experiments on magnetic reconnection. Stenzel and Gekelman in 1981, carried out a detailed laboratory magnetic reconnection experiment. This experiment was carried out applying a linear geometry and an electron magnetohydrodynamic regime, and by considering the ions unmagnetized. Since then, substantial progress has been made in laboratory magnetic reconnection physics (e.g., Yamada et al. 1990, Ono et al. 1996, Yamada et al. 1997 b, Hantao Ji, 1998).

\subsection{Laboratory Studies:}

The investigation of magnetic reconnection in laboratory plasma goes back to the 1960s (Zweibel and Yamada 2009). Initially, the pinch-type experiment for reconnection in the laboratory was conducted for a duration of a few microseconds(Baum and Bratenahl 1974, Syrovastskii et al. 1973, Frank. 1974). These experiments helped to identify the anomalous resistivity in less collisional plasma (Bratenahl and Yeates 1970). An important experiment to study wave related physics in reconnection was conducted by Stenzel and Gekelman (1979); This experiment led to establishing a relationship between the reconnection rate and wave turbulence.

The magnetic reconnection experiment (MRX) was built in 1995 at Princeton Plasma Laboratory (Yamada et al. 1997 a, b). It was used to experimentally verify the Hall effect during magnetic reconnection by observing an out of plane quadrupole magnetic field (Ren et al. 2005). Based on their experimental results, they concluded that the Hall effect which is large in collisionless plasmas is also sufficient for balancing the diffusion regime reconnection electric field (Ren et al. 2005).

In laboratory plasma, under the framework of MRX, considering the collisionality to be variable, two-fluid effects were investigated by Yamada et al. (2005). This experiment was carried out in a regime with a much-reduced electron-ion collision rate. They observed an out-of-plane quadrupole magnetic field component as evidence of the presence of two-fluid effects. Magnetic reconnection has been studied in sawtooth relaxation (Wesson 1987, Kadomtsev 1975, Nagayama 1991, Yamada et al. 1994, Soltwisch 1988, Levinton et al. 1993, Yamada et al. 1994, Nagayama et al. 1991, 1996); and in Reversed Field Pinch and Spheromak Plasmas (Ji et al. 1994, Sarff et al. 2005, Den Hartog et al. 2007, Balbus \& Hawley 1998, Knox et al. 1986, Ono et al. 1988).

These MRX experiments were not aimed to simulate solar flares or other transient activities but instead to provide key data for understanding the fundamental process of magnetic reconnection (for more details please see Zweibel and Yamada 2009). MRX has contributed significantly to laboratory plasma research. It not only allows one to study the coupling among local microscale characteristics of the reconnection layer but also look for global properties such as an external driving force and the evolution of equilibrium in the plasma. Later, by making a controlled prototype of the reconnection layer, some dedicated 
experiments were conducted for magnetic reconnection and their objective was to understand the fundamental process beneath magnetic reconnection (Zweibel and Yamada 2009).

Complimentary, the Versatile Toroidal Facility (VTF) built at Massachusetts Institute of Technology (Egedal et al. 2000) is used to study the fast magnetic reconnection in presence of a guided magnetic field considering the mean free path of the electron is much larger than the plasma dimensions. MRX and VTF are geometrically the same but VTF wins due to its much stronger guide field.In deep terrestrial magnetotail reconnection processes, in situ measurements of electron phase distribution have been interpreted using the theoretical understanding gained from research on reconnection obtained by the VTF (Egedal et al. 2003).

Colliding plasma experiments have also been conducted for studying local and global MHD physics of magnetic reconnection (Yamada et al. 1990, Ono et al. 1993). Laboratory plasma reconnection research has revealed some important findings such as two-fluid effects and reconnection dynamics, identification of electron diffusive layer, heating of ions during experiments of plasma merging and heating of ions during reverse field pinch and the laboratory data has been used for astrophysical scaling with respect to the collisionality. Reconnection has also been studied in the Laser-driven plasmas (Fox et al. 2011, Rosenberg et al. 2014, 2015, Fiksel et al. 2014, Joglekar et al. 2014, Zhong et al. 2010, Nilson et al. 2006, Li et al. 2007)

\subsection{Numerical Studies:}

Magnetic reconnection can convert magnetic energy to heat via ohmic dissipation, convert magnetic energy to bulk kinetic energy and acceleration of plasma, give rise to turbulence, current filamentation and shock waves, accelerate fast particles, and by changing the field line connections, the process may affect the flux of fast particles. Resistive MHD with classical ohmic dissipation is employed to model magnetic reconnection in the interior of the Sun as well as in the lower solar atmosphere.

For providing a fluid description, the plasma should be close to the thermodynamic equilibrium which does not apply in the outer solar atmosphere. Considering temperature and density to be $\mathrm{T}=10^{3} \mathrm{~K}$ and $\mathrm{n}=10^{16} \mathrm{~m}^{-3}$, respectively, the mean free path of a particle comes to be $1 \mathrm{~km}$ in the chromosphere which increases to $1 \mathrm{~mm}$ in the corona for temperature $\mathrm{T}=10^{5} \mathrm{~K}$, and density $\mathrm{n}=10^{7} \mathrm{~m}^{-3}$ ). This mean free path further increases and become $10 \mathrm{~mm}$ at a distance of $1 \mathrm{AU}$ for temperature $\mathrm{T}=10^{5} \mathrm{~K}$ and density $\mathrm{n}=10^{7} \mathrm{~m}^{-3}$. Moving beyond the simple fluid picture has been described in detail by Marsch (2006) and Mayer and Vernet (2007).

Although the two-fluid picture can help to explain the observations, ultimately a more complex kinetic picture is needed. This can help in treating particles and non-equilibrium plasmas. Employing this, the distribution of particles and heat flux can be calculated instead of imposing their forms in an ad-hoc way. The distribution of the particles is very much different from a Maxwellian distribution and shows the presence of particles beams and variations in temperature (Priest 2014). Hence, for modelling magnetic reconnection in the outer solar corona, it is suitable to employ either the kinetic model or two-fluid approach with Hall MHD (Brin and Priest 2007, Priest 2014, Gonzalez 2016)

Though some textbooks exist that provide a good introduction to magnetic reconnection (e.g., Priest 1982, Sturrock 1994, Priest and Forbes 2000, Somov 2000, Tajima and Shibata 2002), few review articles have been written to enhance the existing understanding and knowledge of magnetic reconnection in astrophysical as well as in laboratory plasma (e.g., Yamada 2010,
Zweibel and Yamada2009, 2016, Pontin 2011,Cassak et al. 2017). The focus of the present review article is on two-dimensional models proposed for understanding magnetic reconnection in the outer solar coronal plasma. In Section 2 the Sweet-Parker steady two-dimensional slow reconnection model is presented followed by the Petscheck Mechanism fast reconnection model. Examples of observational evidence for magnetic reconnection based on space-based data are given in Section 3. The article ends with a conclusion. Quantities and formulae that are useful for deriving magnetic reconnection parameters (opted from Zweibel and Yamada 2009) and referred to in this review article are given in Table 1.

\section{Steady Two-Dimensional Reconnection:}

There are some dynamic boundary conditions to be applied when modelling the solar corona: (a) There exists a solar dynamo in the interior of the Sun that operates at the bottom of the convection zone for continuous generation of the magnetic flux. (b) This magnetic flux comes up with the help of buoyancy and comes out through the photosphere to the corona. (c) As the solar rotation is differential, on every rotation the differential rotation and the convective motion wrap the coronal magnetic field and in order to avoid the excessive magnetic stress, the connectivity with the interplanetary fields keeps on breaking up continuously (Aschwanden 2004). Because of these three boundary conditions, coronal fields suffer continuous stress and their adjustment occurs by restructuring their large magnetic fields through topological changes known as magnetic reconnection processes (Aschwanden 2004). Whenever the magnetic stress builds up, the restructuring of the magnetic field takes place. These topological changes cause the liberation of free, non-potential energy and this energy further results in heating the plasma, accelerating the particles and contributing to the kinematics of coronal plasma. Although magnetic reconnection can take place in a slow manner, it can still contribute to coronal heating, but mostly it takes place in a violent manner and is observed in the form of solar flares or CMEs.

Quasi-steady magnetic reconnection has been proposed as a direct plasma heating mechanism. In this kind of magnetic field reconnection process, the coronal plasma dissipates the magnetic energy (Parker 1963a, 1972, 1979, 1983; Sturrock and Uchida 1981, VanBallegooijen 1986). It has also been suggested that this type of reconnection supplies plasma heating directly to solar flares (e.g., Sweet 1958, Parker 1963a, Petschek 1964, Carmichael 1964, Sturrock 1966). The present concept has been used in many theoretical models to understand coronal heating and solar flares. The forward movement of a new magnetic flux system towards an old subsisting magnetic flux system forms a layer and the magnetic fields can then be directed in opposite directions at both sides of the boundary. To maintain a continuous rearrangement from - to + polarity, the magnetic field has to drop to zero at this borderline. In that case, the balance between the magnetic and thermal pressure in the neutral boundary is given as $B_{1}^{2} / 8 \pi+p_{1}=p_{\text {neutral }}+B_{2}^{2} / 8 \pi$ (Aschwanden 2004). This relation provides a higher thermal pressure $p_{\text {neutral }}$ with the magnetic field $\mathrm{B}=0$ inside the neutral layer where $\mathrm{B}_{1}, \mathrm{~B}_{2}$ are the magnetic fields on both sides of the layer.

Here the focus is placed on the steady-state MHD mechanism for slow and fast magnetic reconnection. The Sweet-Parker Model is presented as an example for slow magnetic reconnection in section 2.1 and the Petscheck Mechanism for fast magnetic reconnection in section 2.2 . 
Table 1: Some quantities, along with their formulae and values in cgs unit, that are useful for deriving parameters of magnetic reconnection (opted from Zweibel and Yamada 2009).

\begin{tabular}{|l|l|l|l|}
\hline Symbols & Quantity & Formula & Value \\
\hline$\tau_{e}$ & Electron Collision time & $3 / \sqrt{32 \pi}\left(m_{e}^{1 / 2}\left(k T_{e}\right)^{3 / 2} / \lambda e^{4} n_{i}\right)$ & $2.9 \times 10^{-2} /(\lambda / 10) T_{e}^{3 / 2} n_{e}^{-1} s$ \\
\hline$\omega_{c e}$ & Electron gyrofrequency & $e B / m_{e} c$ & $1.8 \times 10^{7} B s^{-1}$ \\
\hline$\omega_{c p}$ & proton gyrofrequency & $e B / m_{p} c$ & $9.6 \times 10^{3} B s^{-1}$ \\
\hline$\omega_{p e}$ & Electron plasma frequency & $\left(4 \pi n_{e} e^{2} / m_{e}\right)^{1 / 2}$ & $5.6 \times 10^{4} n_{e}^{1 / 2} s$ \\
\hline$\omega_{p p}$ & proton plasma frequency & $\left(4 \pi n_{e} e^{2} / m_{p}\right)^{1 / 2}$ & $1.3 \times 10^{3} n_{e}^{1 / 2} \mathrm{~s}$ \\
\hline$\delta_{e}$ & Electron skin depth & $c / \omega p e$ & $5.4 \times 10^{5} n_{e}^{-1 / 2} \mathrm{~cm}$ \\
\hline$\delta_{p}$ & proton skin depth & $c / \omega p p$ & $2.3 \times 10^{7} n_{p}^{-1 / 2} \mathrm{~cm}$ \\
\hline$\sigma$ & Electrical conductivity & $\omega_{p e}^{2} \tau_{e}^{/ 4 \pi}$ & $7.3 \times 10^{6} T_{e}^{3 / 2}(10 / \lambda) s$ \\
\hline$\eta$ & Magnetic diffusivity & $c^{2} / 4 \pi \sigma=\delta_{e}^{2} / \tau_{e}$ & $9.9 \times 10^{12} / T_{e}^{3 / 2}(10 / \lambda) s$ \\
\hline$v_{A}$ & Alfvén speed & $B / \sqrt{4 \pi m_{p} m_{e}}=\omega_{c i} \delta_{i}$ & $2.2 \times 10^{11} \mathrm{Bn}_{p}^{-1 / 2} \mathrm{~cm} \mathrm{~s}$ \\
\hline$S$ & Lundquist number & $L v_{A} / \eta^{-1} L / \delta_{i}\left(\omega_{c e} \tau_{e}\right)$ & $2.3 \times 10^{-2} \mathrm{LBT}_{e}^{3 / 2} n_{e}^{-1 / 2}(\lambda / 10)^{-1}$ \\
\hline
\end{tabular}

\subsection{Sweet-Parker Model:}

The rate of magnetic reconnection is the speed of field lines, as they reach the reconnection site. For slow two-dimensional magnetic reconnection, the Sweet-Parker model is widely accepted. Sweet and Parker assumed that the diffusion layer is steady and spread in the entire area among two oppositely directed magnetic fields (Sweet 1958 a, b, Parker 1957, 1963b). They considered a diffusion layer with a length of say $2 \mathrm{~L}$ and a width 21 , and the layer to be situated between oppositely-directed magnetic field lines (see Figure 1). The length of this layer is considered as the global external length-scale $\left(L_{e}\right)$. Ohm's Law, the equation of motion and equation of continuity remain the main ingredients while constructing this theoretical model.

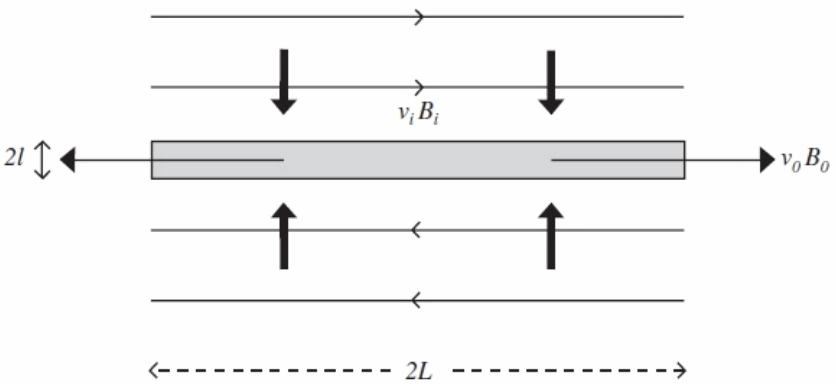

Figure. 1 Sweet-Parker reconnection. The shaded region represents the diffusion region. The light and solid head arrows represent the velocity of magnetic field lines and plasma, respectively (Opted from Priest 2014).

If a magnetic field $B_{\mathrm{i}}$ is entering the diffusion layer and diffusing outwards from the diffusion layer at the same speed $\mathrm{v}_{\mathrm{i}}$, then $v_{\mathrm{i}}$ can be given as $v_{\mathrm{i}}=\eta / l$. Being mass conserved, the rate at which the mass will enter $\left(4 \rho L v_{\mathrm{i}}\right)$ from both ends will be equal to the rate $\left(4 \rho l v_{0}\right)$ at which the mass will leave both ends at a velocity $v_{0}$. Hence, if the density is uniform, $L v_{\mathrm{i}}=l v_{0}$. These two relations allow one to calculate the inflow speed to be $v_{\mathrm{i}}=\left(\eta v_{0} / L\right)^{1 / 2}$. The magnetic reconnection rate, in dimensionless parameters, is given as $M_{i}=\left(v_{0} / v_{\mathrm{Ai}}\right)^{1 / 2} /\left(R_{m i}\right)^{1 / 2}$, where $M_{i} \equiv v_{i} / v_{A i}$ is the inflow Alfven
Mach number or dimensionless reconnection rate and $R_{m i}$ is the magnetic Reynolds number(Priest 2014). Knowing the outflow speed $v_{0}$ for a given length $L$, one can calculate the inflow speed $v_{\mathrm{i}}$ and then calculate (a) the width of the sheet to be $l=L v_{\mathrm{i}} / v_{0}$ and (b) the strength of outflow magnetic field $\left(B_{0}\right)$ using the conservation of flux $\left(B_{i} v_{i}=B_{0} v_{0}\right)$ (Priest 2014). The outflow speed $v_{0}$ estimated is given as $v_{0}=B_{i} /(\mu \rho)^{1 / 2} \equiv v_{A i}$, here, $v_{A i}$ s the inflow Alfven speed and the speed of reconnection of fields is given as $v_{i} v_{A i} /\left(R_{m i}\right)^{1 / 2}$. The plasma being ejected from the sheet of width $l=L /\left(R_{m i}\right)^{1 / 2}$ at an outflow speed of $v_{0}$ equals the Alfven inflow speed $v_{A i}$ and the magnetic strength of the outflow magnetic field is given by $B_{0}=B_{i} /\left(R_{m i}\right)^{1 / 2}$. Being $R_{m i}>>1$, from the above three relations for $v_{i}, l$ and $B_{0}$, yields, $v_{\mathrm{i}}<<v_{A i}, B_{0} \ll B_{i}$ and $l<<L$. The reconnection rate in the Sweet-Parker mechanism is given as $M_{i}=\left(R_{m i}\right)^{-1 / 2}$. The length $\mathrm{L}$ and magnetic Reynolds number $\left(R_{m i}\right)$ in the Sweet-Parker mechanism are identified as the global external length scale and global magnetic Reynolds number, respectively. The Sweet-Parker model has a reconnection rate $\mathrm{v}_{\mathrm{i}}=\mathrm{v}_{\mathrm{Ai}} /\left(R_{\mathrm{m}}\right)^{1 / 2}$, where $\mathrm{v}_{\mathrm{Ai}}$ is the inflow Alfven speed, $R_{\mathrm{m}}$ is the magnetic Reynolds number given as $R_{\mathrm{m}}=L v_{A i} / \eta$ (Priest 2014) and $\mathrm{L}$ is the length of the sheet. In the solar corona, the global magnetic Reynolds number varies between $10^{6}-10^{12}$ and hence the reconnection rate yields to be of the order of $10^{-3}-10^{-6}$ of the Alfven speed. Therefore, in the case of solar plasma eruptions, the Sweet-Parker model reconnection rate is too slow for explaining the rapid energy release of solar flares.

\subsection{Petscheck Mechanism:}

Fast magnetic reconnection simply means a reconnection rate much higher than the Sweet-Parker rate of reconnection. With the discovery that the Sweet-Parker mechanism was too slow to account for the rapid energy release in solar flares, there was a hunger for finding an alternative mechanism. At a conference on physics of solar flares (AAS-NASA Symposium held 28-30 October 1963 at the Goddard Space Flight Center, Greenbelt, MD),Petscheck (1964)came up with a masterstroke solution and immediately Gene Parker who was in audience asserted that this was the solution that he was looking for(Tsinganos 1996). To resolve the slowness of the Sweet-Parker reconnection rate, 
Petscheck in 1964 suggested the key idea of decreasing the length of the diffusion region much lower than the global external length scale (i.e., $L<<L_{e}$ ) (Petscheck 1964). The assumption was that if the diffusion region is small then all the fluid will not pass through it but instead will be redirected by the standing shock waves. The Petscheck model has been well summarised in the literature (e.g., Priest 1982, Jardine 1991, Priest and Forbes 2000, Treumann and Baumjohann 1997, Tajima and Shibata 2002). The decrease in the length of the current sheet results in shortened propagation time through the diffusion region and thus a faster reconnection process (Aschwanden 2004).

Petschek further suggested that the slow-mode shock waves behave as the obstacle for the flow which is supersonic relative to the slow-mode shock waves. These shocks are generated in the same way that hydrodynamical shocks are generated during an aeroplane propagating at supersonic speed (Tsinganos 1996). Petscheck realized that magnetic energy can also be converted into heat and kinetic energy via slow-mode shock waves. He proposed that as the magnetic energy propagates in the medium with a given speed the slow-mode shock waves will propagate from the diffusion region and stand in the flow. However, if the magnetic field is ahead of a shock and directed along the normal it will keep the tangential component in switch on mode and such a fast shock is called a switch on shock. The slow-mode shocks, under conditions of switch-off limit, travel at the Alfv'enic speed depending on the normal field (Priest 2014)), travel in the medium at rest with a speed $v_{s}=B_{N} / \sqrt{\mu \rho}$, where $B_{N}$ is the magnetic field normal to the front of the shock and $v_{\mathrm{s}}$ is said to be the Alfven velocity which depends on $B_{N}$. It twists the magnetic towards the normal and results in a decrease of its strength (Priest 2014, Tsinganos 1996 ) (shown in figure-2). It simultaneously heats the plasma by accelerating it to a velocity which equals the Alfven velocity $\left(v_{A}\right)$ (Tsinganos 1996). These shocks serve as the main sites for energy conversion. Two-fifths of the inflowing magnetic energy converts to heat while three-fifths of it converts to kinetic energy (Tsinganos 1996). Now, if the upstream plasma moves with a speed $v_{\mathrm{s}}$ (the speed of moving shocks), the shocks will be stationary. The model of Petschek is considered to be "Almost Uniform Reconnection" because the inflow region is a current free magnetic field that only results in a small perturbation to the uniform magnetic field $B_{e} \hat{x}$ with $M_{\mathrm{e}} \ll<1$ (Tsinganos 1996, Priest 2014).

When the magnetic field approaches the diffusion region, the value of the magnetic field $B_{e}$ decreases to $B_{i}$ which can be obtained as- $B_{i}=B_{e}\left[1-\left(4 M_{e} / \pi\right) \log \left(L_{e} / L\right)\right]$. Petscheck (1964)calculated the maximum rate of reconnection $\left(M_{e}^{*}\right)$ by considering that the magnetic field value becomes half at a distance of $L_{e}$ (i.e., $B_{i}=0.5^{*} B_{e}$ ), respectively. Hence, the reconnection rate is dependent on $\log \left(R_{m e}\right)$ where $R_{m e}=L_{e} v_{A e} / \eta$. As per the conditions of the solar corona, the Reynolds number is very high and lies in a range of $10^{8}-10^{12}$ which yields the reconnection rate for the Petscheck mechanism to be in the range of 0.01-0.02 (Aschwanden 2004). This also yields an inflow speed of $10-20 \mathrm{~km} \mathrm{~s}^{-1}$ (estimated as $v_{A} * M_{e}^{*}$, where a typical value of the Alfven speed is $1000 \mathrm{~km} \mathrm{~s}^{-1}$ for solar coronal conditions) (Aschwanden 2004).

With the discovery of self-similar solutions of the external regions (Soward and Priest 1977), Petscheck's mechanism, having four standing slow mode shock waves which extend from the small central current sheet, was widely accepted as a mechanism suitable to explain fast reconnection (Petscheck 1964). Additionally, it was fast enough to handle rapid solar flare energy release. It was the year of 1986 that some resistive MHD and theoretical models, and numerical experiments came into existence. They catalysed the process of furthering our understanding of magnetic reconnection. The solutions of the numerical experiments carried out by Biskamp (1986) were very different from those of Petscheck's and hence put a question mark on the legitimacy of Petscheck's mechanism. This discrepancy in both solutions was resolved by Priest and Forbes (1986) by realizing that boundary conditions enforced by Biskamp (1986) were different from Petscheck's. High-resolution numerical simulations developed during the last decade have reasonably improved our understanding of fast magnetic reconnection by demonstrating the break-up of the current sheets into magnetic islands. These numerical simulations also show that the reconnection rate becomes independent or weakly dependent on the Lundquist number challenging the hypothesis of Petscheck's mechanism of having slow-mode shocks (Bhattacharjee et al. 2009, Loureiro et al. 2012, Huang and Bhattacharjee 2010, 2013).

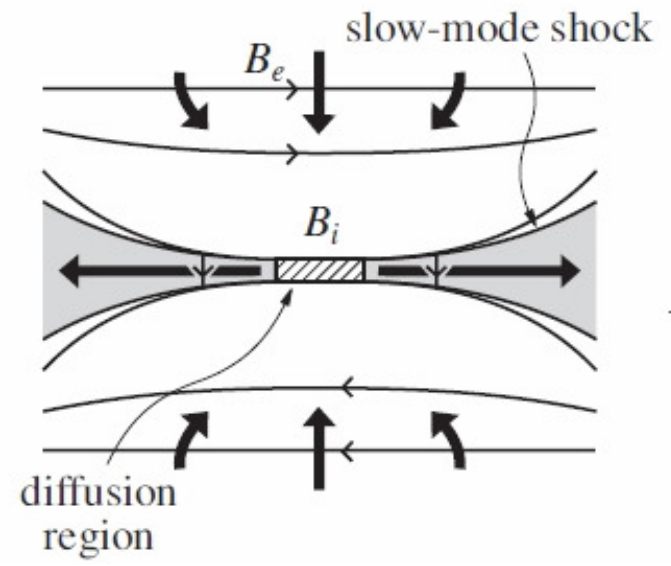

Figure. 2 The Petscheck model. The diffusion region is displayed by the central shaded area. The other two shaded areas represent the plasma which is heated and accelerated by the shocks (Opted from Priest 2014).

\section{Observational evidence of Magnetic Reconnection in the Solar atmosphere}

In the early years, solar flares were identified as a sudden brightening in the solar chromosphere and visible only in H-alpha wavelength. The existence of the positive and negative polarity of the magnetic field and formation of closed loops by them were only discovered after measurements of the line of site magnetic fields in the photosphere by means of the Zeman effect (Zeeman 1897). There exist some observational evidence for some of the signatures of magnetic reconnections (e.g., cusp-like hot structures (Tsuneta et al. 1992), loop top hard x-ray sources (Masuda et al. 1994, Sui and Holman 2003), reconnection inflows and outflows (Yokoyama et al. 2001, Li and Zhang 2009, Su et al. 2013, Sun et al. 2015, Yang et al. 2015, Asai et al. 2004, Savage et al. 2010, Liu et al. 2013, Chen et al. 2016), observations of current sheets (Webb et al. 2003, Lin et al. 2005, Liu et al. 2010, Xue et al. 2016, Yan et al. 2018), ejection of the plasmoids (Shibata et al. 1995, Nishizuka et al. 2010, Takasao et al. 2012) and the loop-loop interactions (Sakai and de Jager 1996, Li et al. 2014))

It is well known that at very high temperatures (of the order of $\left.10^{8}-10^{10} \mathrm{~K}\right) \mathrm{H}$-alpha emissions occur with X-rays, gamma-rays and emission of $\mu$-waves. The origin of these accompanied emissions is associated with the excited electrons (e.g., electron's bremsstrahlung and gyro-synchrotron radiation at higher energies give the hard $\mathrm{X}$-rays and the emission of $\mu$-waves). However, gamma-rays are an outcome of the annihilation of electrons and positrons, neutron capture on protons (for more details see Weller and Roberson 1980) and also generated during the transitions from a high energy state to low energy state (gamma decay) (Zweibel et al. 2009).In the modern era, multi-wavelength observations obtained by high spatial and temporal resolution detectors onboard spacecraft (e.g., Yohkoh, SoHo, Trace, RHESSI, Hinode and SDO etc.) have enabled us to improve our understanding of solar eruptions and the physics behind them. 
Using Yohkoh Soft X-ray Telescope (SXT) data, Tsuneta et al. (1992) observed a soft X-ray flare at the solar limb from its pre-flare to post-flare stage. They observed a "helmet streamer" like an arch that formed hours before the solar flare with the expansion of the active region magnetic field. They stated that the flaring started gradually with the expansion. Hence, this expansion probably triggered the flare. This arch further started to flare and was observed to increase in height. A footpoint separation was also observed at a velocity of $10-30 \mathrm{~km} \mathrm{~s}^{-1}$. Based on the morphology of the arch-flare, they suggested that the formation of the current sheet was the primary source of the flare. They further analyzed the temperature structure of the X-ray loop that indicated an ongoing reconnection process near the top of the loop supplied the flare energy.

Masuda et al. (1994), using the observations from the Yohkoh, Hard X-ray Telescope (HXT) and SXT, reported the observation of a compact flare which is geometrically analogous to that of a two-ribbon flare. They observed a hard X-ray source near the limb and above the soft X-ray loop. They suggested that magnetic reconnection is taking place above the loop. Further, in a succeeding work, Shibata et al. 1995, searched for a plasma ejection using Yohkoh/SXT data in eight compact loop flares including the Masuda flares. Their observations revealed that all the flares were accompanied by X-ray plasma ejections. These plasma ejections were observed above the soft X-ray coronal loop with an ejection speed in the range $50-400 \mathrm{~km} \mathrm{~s}^{-1}$. The findings of Shibata et al (1995) further support the hypothesis of magnetic reconnection in impulsive compact loop flares. Tsuneta (1996) analyzed the temperature structure of a prototypical flare, which occurred on 21 February 1992. They measured the reconnection point situated at a height of $8-18 \times 10^{4}$ kilometres above the top of a flare loop. They estimated the inflow speed to be $56 \mathrm{~km} \mathrm{~s}^{-1}$ (Alfven Mach number 0.07). With SXT/Yohkoh, Tsuneta (1997) observed a plasmoid like feature that occurred near the limb on 2 December 1991. He observed a rising loop 10 minutes before the flare. This rising loop further evolved to a plasmoid-like structure during the impulsive phase of the flare. He estimated the speed of the rising loop to be $96 \mathrm{~km} \mathrm{~s}^{-1}$. An X-shaped feature was observed, formed below the X-point and a bright X-ray loop formed under the $\mathrm{X}$-point. This $\mathrm{X}$-shaped feature specifies a magnetic neutral point with a large-scale magnetic separatrix structure. Inverse Vshaped high-temperature edges observed above the soft X-ray loop and below the $\mathrm{X}$-point were interpreted as slow shocks heated reconnected loops. Yokoyama et al. (2001) studied a flare that occurred on 18 March 1999. They observed this flare on the northeast limb accompanied by a cusp-shaped soft X-ray loop and plasmoid ejection. In the EUV wavelength of the same flare, they observed an ejection of a "bubble-like void". With the ejection of the void, they observed magnetic reconnection occurring at the disconnecting point. They also observed that movement of the pattern towards the magnetic X-point with a speed of $5 \mathrm{~km} \mathrm{~s}^{-1}$ to be the upper limit of reconnection.

A flare occurring on the north-west limb (15 April 2002), showing a cusp-shaped flare loop in the rising phase, was observed by Sui and Holman (2003)using the RamatyHighEnergySolarSpectroscopicImager. They observed a current sheet formed during this flare event. At the beginning of the impulsive rise phase, the cusp-shaped feature detached from the underlying flare loop remained stationary for 2 minutes. A simultaneous shrinking in the underlying flare loops had been noticed with a speed of approximately $9 \mathrm{~km} \mathrm{~s}^{-1}$. As they observed an increment in the temperature of the underlying loop and coronal source towards higher altitudes and lower altitudes, respectively, they proposed a possible formation of the current sheet between the top of the flare loop and coronal source, during the early impulsive phase. They also observed evidence for a possible expansion of the current sheet in the upward direction.

Lin et al (2005) have reported direct observations of magnetic reconnection in an eruption dated 18 November 2003. They used spaceborne as well as ground-based observations from the EUV Imaging Telescope, Ultraviolet Coronagraph Spectrometer, and Large Angle Spectrometric Coronagraph experiment onboard the Solar and Heliospheric Observatory, the Reuven Ramaty High Energy Solar Spectroscopic Imager, and the Mauna Loa Solar Observatory Mark IVK-Coronameter. This eruption began with the rapid expansion of magnetic arcades on the east limb of the Sun, which further led to the development of an energetic Halo CME, a long current sheet and a bunch of bright flare loops. They observed that the leading edge of CME and the core were moving with a maximum speed of 1939 and $1484 \mathrm{~km} \mathrm{~s}^{-1}$, respectively. The average inflow velocities observed near the current sheet were found to be in the range of $10.5-106 \mathrm{~km} \mathrm{~s}^{-1}$ however, the outflow velocities in the range $460-1075 \mathrm{~km} \mathrm{~s}^{-1}$.

Wang et al. (2007) reported observations of fast magnetic reconnection outflows in the solar corona, using SoHO Solar Ultraviolet Measurements of Emitted Radiation (SUMER) data. They measured the outflow reconnection speed being in the range $900-3500 \mathrm{~km} \mathrm{~s}^{-1}$ and the magnetic field estimated by them near the reconnection region was 19-37 Gauss.

$\mathrm{Li}$ and Zhang (2009) observed an M class flare in multiwavelength that occurred on 23 March 2000. They observed sidewards motions of two EUV loops over a hard X-ray source with velocities of 75 and $25.6 \mathrm{~km} \mathrm{~s}^{-1}$, respectively. They considered these motions as, reconnection inflow. During the second motion, they observed an X-shaped feature over the postflare loop. They also noticed two separated flare ribbons associated with these motions with average velocities of 3.3 and $1.3 \mathrm{~km} \mathrm{~s}^{-1}$. The consistency of the sideward motions and the separations with two peaks of the X-ray flux specifies two types of reconnection in the flare. For these two reconnection processes, they estimated the corresponding coronal magnetic field strength to be 13.2-15.2 Gauss and the reconnection rate to be 0.05 and 0.02 , respectively. Their observations also revealed the bidirectional movement of bright points, hypothesized as ejected plasmoids with upward and downward speeds 45.4-556.7 $\mathrm{km} \mathrm{s}^{-1}$, possibly ejected when the magnetic reconnection occurred.

The observations of Liu et al. (2010) are very important as they provide a comprehensive view of magnetic reconnection in the solar corona by observing evidence of magnetic reconnection at several locations within one event. They observed plasma inflow and outflow along with expanding cusp-shaped dimming as well as chromospheric evaporations and radio emissions, as the favourable evidence for the process of magnetic reconnection in the solar corona. They observed an elongated sheet-like structure with $>0.25 \mathrm{R}$ and (5-10) $\times 10^{3}$ kilometres as its global length and macroscopic width, above the cusp like flaring loop in the rising phase of the flare. They proposed that this EUV elongated feature is a Y-shaped current sheet.

Plasma voids travelling towards the Sun, occasionally observed in the outer atmosphere of the Sun or in the corona during solar flares are called supra-arcade downflows (SADs). Their first description was credited to SXT/Yohkoh in 1999 (McKenzie and Hudson 1999). Since then, SADs have continuously been observed by TRACE, SOHO/LASCO, SOHO/ SUMER, Hinode/XRT. Observations of SADs suggest that they are the cross-sections of thin flux tubes renounced from the site of reconnection in the corona.

There exists evidence of observations of SADLs (SAD loops), under similar circumstances. These features are theoretically interpreted as the perpendicular view of supra-arcade downflows. Since SADs and SADLs cannot be observed in three dimensions by an independent imaging instrument, this connection is considered as a hypothetical connection. If SADs are viewed from an angle not close to the perpendicular to arcade axis, the downflows appear as shrinking loops. Savage and McKenzie (2011) observed SADs in eruptive solar flares and derived their physical parameters (e.g., velocity, acceleration, area, magnetic flux, shrinkage energy and reconnection rate etc). They measured 
the cross-sectional area $\left(\sim 2-90 \mathrm{Mm}^{2}\right)$ and the speed of the flows $\left(10^{2} \mathrm{~km} \mathrm{~s}^{-1}\right)$ with the accelerations near to zero or decelerating. They measured to possess a path length of $\sim 10^{4} \mathrm{~km}$. Each tube was found to carry a flux of $\sim 10^{18} \mathrm{Mx}$ and release $10^{27} \mathrm{ergs}$ of energy.

Using UVCS data, in the Fexviii line at a temperature of approx $10^{6.8} \mathrm{~K}$, Ciaravella and Raymond (2008) observed a current sheet accompanied with four CMEs. This was the first Ultraviolet observation of the evolution of the current sheet from its onset. Takasao et al. (2012) analyzed the EUV data of AIA/SDO corresponding to a flare that occurred on 18 August 2010 and they reported simultaneous observations of reconnection inflow and outflow. They observed some plasma blobs in the sheet during the rising phase of the flare, which further ejected bi-directionally along the sheet structure (i.e., outflow). At the same time, threads were observed moving towards the sheet structure (i.e., inflow). They estimated the upward ejecting speed of blobs to be in the range $220-460 \mathrm{~km} \mathrm{~s}^{-1}$ and downwardly ejected blob's speed in the range $250-280 \mathrm{~km} \mathrm{~s}^{-1}$. They also noticed an alteration of inflow speed from 90 to $120 \mathrm{~km} \mathrm{~s}^{-1}$, over a time of 5 minutes. They estimated the reconnection rate and found it to vary in range 0.20 0.55 during this time. They observed the collision and merging of blobs with each other before their ejection from the sheet-like feature. They further suggested a possibility that these blobs can be plasmoids and the sheet-like structure can be the current sheet.

Using Hinode/XRT and STEREO-B/EUVI data on 16 March 2007, Zhang et al. (2012) studied two coronal bright points (hereafter CBPs). They observed gentle brightening as well as CBP flashing. They noticed the occurrence of CBP flashes with the SXR jets and observed that the CBPs covered with a domelike separatrix surface were accompanied with a null point above. They suggested the CBP flashing was caused by a null point magnetic reconnection and the gentle brightening because of interchange reconnection along the separatrix surface. In a CBP observation ( 20 minutes) captured by AIA/SDO on 05 March 2011, Ning and Guo (2014) observed some moving structures. This CBP was found to be bright with a curved shape along a magnetic loop connecting negative and positive magnetic fields. The observations reveal an intermittent movement of many bright structures along the loop legs towards the two foot-points from the bright core of the CBP. They interpreted them to be the outflows (average speed $380 \mathrm{~km} \mathrm{~s}^{-1}$ with a period of $80-100 \mathrm{~s}$ ) generated after magnetic reconnection, which took place during the CBP.

Innes et al. (2015) obtained a survey of line profiles produced by small-scale events, considered as reconnection sites. Their observations suggested the effectiveness of plasmoid instability rather than Petscheck's mechanism in the small-scale magnetic reconnection. Yang et al. (2015), using H-alpha data observed from NVST (New Vacuum Solar Telescope)reported observational evidence of magnetic reconnection between two sets of small-scale and anti-parallel loops with an X-shaped topology. The magnetic reconnection took place in two steps. In the first step, a gradual reconnection took place between two sets of antiparallel loops and new loops were formed which stacked together As this reconnection lasts for a few tens of minutes, it is considered to be slow reconnection. In the second step, when the anti-parallel loops approached each other very quickly, former loops disappeared. This magnetic reconnection considered to be rapid reconnection as it lasts for three minutes.

The reconnection of an erupting filament and the loops of its vicinity was studied by $\mathrm{Li}$ et al. (2016). An X-type feature formed where the erupting filament encountered the loops. Later, the loop became straight and a bright current sheet formed at the interfaces The plasmoids appeared in the current sheet and were observed to have bidirectional propagation. Their results presented evidence for the theory of three-dimensional reconnection.

More recently, $\mathrm{Li}$ et al. (2018) reported the formation of the current sheet in a solar flare that occurred on 10 September 2017. They used data from AIA/SDO and EIS/Hinode instruments for their analysis. Their observations revealed that the current sheet was mainly visible at high temperatures (i.e. > $10 \mathrm{MK}$ ), especially in the Fe XXIV $192.03 \AA$ line. The formation temperature of $\mathrm{Fe}$ XXIV $192.03 \AA$ line is approximately $18 \mathrm{MK}$ and it shows nonthermal motions of velocities (up to $200 \mathrm{~km} \mathrm{~s}^{-1}$ ) in the current sheet. The existence of this particular line suggests the presence of turbulent motions in the current sheet. They estimated the thickness of the current sheet to be 7-11 Mm.

Yang et al. (2018) observed an X class flare associated with a CME that occurred at the west limb of the Sun on 10 September 2017. They reported simultaneous observations of inflow, current sheet formation and a cusp-shaped feature that took place after a flux rope eruption. During the final stage of the eruption, they observed the separation of the current sheet in several small current sheets. They measured the width of the current sheet to be $10^{3}$ kilometres.

$\mathrm{Li}$ et al. (2018) observed some small-scale reconnection events via spectroscopic and imaging observation of IRIS and AIA/SDO. The event they observed and identified as the IRIS jets in the chromosphere and transition region possessed a close association with flux cancellation in the photosphere and EUV brightening in the corona. As the IRIS jets were detected in the Mg II k, C II and $\mathrm{Si}$ IV spectral lines, there is an indication that the reconnection site is from the lower chromospheres to the transition region. The coexistent flux cancellation supports magnetic reconnection and points out the location of the driver of the energy release in the photosphere. They estimated the energy budgets in the solar atmosphere to show that the magnetic energy in the photosphere is sufficient to drive the IRIS jets in the chromosphere and transition region, further causing the EUV brightening observed in the corona. They estimated the released energy to be $(6.7 \pm 1.9) \times 10^{27}$ ergs) and the upper limit value of kinetic energy in the transition region from the IRIS jets $\left(2.6 \times 10^{26}\right.$ ergs) along with the upper limit of the thermal energy in the corona by the EUV brightening $\left(7 \times 10^{24}\right.$ ergs $)$.

The magnetic reconnection, as well as acceleration of mass in flares and CME events, have been studied by Qiu et al. (2004). Their study contains an X 1.6 and M 1.0 class flare, each accompanied by a fast halo CME. Based on their observations they concluded that magnetic reconnection is related to the high energy flare emissions and rising motions of erupting flux. They found a temporary correlation between magnetic reconnection, non-thermal emissions in hard X-rays and microwaves in the solar corona. They estimated the reconnection electric field $\left(\mathrm{E}_{\mathrm{rec}}\right)$ and rate of magnetic flux change $\left(\varphi_{\text {rec }}\right)$ for both $\mathrm{X}$ and $M$ class flares to be $\sim 5.8 \mathrm{~V} \mathrm{~cm}^{-1}, 3 \times 10^{18} \mathrm{Mx} \mathrm{s}^{-1}$ and $\sim 0.5 \mathrm{~V} \mathrm{~cm}^{-1}, 2 \times 10^{18} \mathrm{Mx} \mathrm{s}^{-1}$, respectively. In addition, they estimated the mass acceleration for both flares to be 2.6 and $0.2-0.4 \mathrm{~km} \mathrm{~s}^{-2}$, respectively.

Wang et al. (2007) observed hot and fast magnetic reconnections outflows for the first time. They exploited the spectroscopic observations of SUMER/SOHO dated April 16, 2002, for examining a flare associated with the NOAA AR 9901, very close to the northwest limb. This was identified as a GOES 2.5 flare which was associated with a slow CME (Goff et al. 2005). As a rise in the reconnection site across the SUMER spectrometer slit was noticed, signatures of blue and redshifts were also observed in Fe XIX line sequence. These signatures reflect the respective up and downflows of hot plasma jets. They interpreted these observed up and downward jets as high-speed outflows from magnetic reconnection sites. The magnetic reconnection site was found to be below and above the slit, while observing down and upflowing jets, respectively. The outflow speed measured by them lies in a range of $\sim 900$ and $3500 \mathrm{~km} \mathrm{~s}^{-1}$. Their finding remained in agreement with the theoretical estimations of the Alfvenic outflows in magnetic reconnection region. They theoretically estimated the magnetic field near reconnection region and found it to lie in a range of $19-37 \mathrm{G}$.

Takasao et al. (2012) examined a Goes C 4.5 class flare in NOAA AR 11099observed by AIA/SDO on 18 August 2010 and 
reported the existence of magnetic reconnection inflow and outflow. They observed some plasma blobs ejected bidirectionally along the sheet-like structure and they interpreted it as outflows. They also noticed some threads moving towards the sheet-like structure and interpreted them as inflows. They found the upward and downward ejection velocities in the range of $220-460 \mathrm{~km} \mathrm{~s}^{-1}$ and $250-280 \mathrm{~km} \mathrm{~s}^{-1}$, respectively. However, the inflow speed found to change from $90 \mathrm{~km} \mathrm{~s}^{-1}$ to $12 \mathrm{~km} \mathrm{~s}^{-1}$ in a time span of 5 minutes. The reconnection rate, they estimated found to vary in a range of 0.20 to 0.055 . They hypothesized this sheet-like structure to be a current sheet and plasma blobs as plasmoids or magnetic islands. They also observed that the plasma blobs were being collided or merged before they were ejected from the sheet-like structure.

Exploiting multiwavelength EUV observations of AIA/SDO and x-ray observations of RHESSI, Su et al. (2013) presented observational evidence of magnetic reconnection in a solar flare. This GOES C 2.3 class flare was observed near the southeast limb on 17 August 2011. They observed the inflow and outflow speeds in the range of $\sim 20-\sim 70 \mathrm{~km} \mathrm{~s}^{-1}$ and $\sim 90-\sim 440 \mathrm{~km} \mathrm{~s}^{-1}$, respectively. Their estimated reconnection rate was found to vary from $\sim 0.05$ to $\sim 0.5$.

Cairns et al (2018) have recently studied magnetic reconnection, and associated type III solar radio bursts and X-ray emissions using spaceborne as well as ground-based observations. The magnetic reconnections have been studied by exploiting the SDO UV-EUV data and type III bursts have been identified in the data observed by the Murchison Widefield Array, the Learmonth radio spectrograph and STEREO B spacecraft. In addition, X-ray emissions have been studied using RHESSI data. They observed strong up and downward pairs of jets along with the current sheets, strong outflows along the pairs of magnetic field lines, cusp-like geometries on top of time-varying magnetic loops as evidence of magnetic reconnection. They found a causal link between the observed type III bursts, X-ray bursts and the specific reconnection events. Interestingly their observations revealed the reconnection sites lying at low heights of 5-10 Mm. Their observations also provide direct evidence that semi-relativistic electrons energized in the magnetic reconnection regions may give rise to type III radio bursts. They emphasise that not all observed magnetic reconnection events produce coronal, interplanetary or $\mathrm{X}$-ray events but the electrons can produce radio, UV, EUV and $\mathrm{X}$-ray bursts if they leave the reconnection sites under some special conditions.

Analyzing EUVI/STEREO and AIA/SDO data on 27 January 2012 (00:00 to 03:00 UT), Sun et al. (2015) reconstructed the three-dimensional magnetic topology of fast reconnection in a solar eruption event and studied the role of reconnection in the flare and CME. They observed the bilateral inflows, downflows related to reconnection outflows and plasma heating near the reconnection site as evidence of reconnection. They tracked several inflow trajectories and the measured velocities of inflows were found to lie within a range of $0.1-3.7 \mathrm{~km} \mathrm{~s}^{-1}$. Every trajectory was noticed to show a tendency of increasing velocity towards the reconnection site. They have also observed downflows in a range of $100-200 \mathrm{~km} \mathrm{~s}^{-1}$.

Analyzing data from New Vacuum Solar Telescope (NVST), Solar Dynamics Observatory (SDO), X-Ray Telescope (XRT)/ Hinode and Soft X-ray Imager (SXI)/GOES, observational evidence of small-scale reconnection triggered by an erupting filament has been reported by Xue et al. (2015). They employed the HMI/SDO magnetograms for examining the 3-dimensional structure of the reconnection region and to exhibiting the change in topology. They observed a current sheet with the length and width to be $4.3 \times 10^{3}$ and $1.06 \times 10^{3} \mathrm{~km}$, respectively. The reconnection inflows of magnitude $3.7-25 \mathrm{~km} \mathrm{~s}^{-1}$ have also been observed on both sides of the current sheet. However, they observed a downward moving reconnection outflow of magnitude $41.7-43.7 \mathrm{~km} \mathrm{~s}^{-1}$ on one side of the current sheet.
Hong et al. (2016) analyzed a GOES-B class flare that had occurred in NOAA AR 12146 on August 24, 2014, and studied magnetic reconnection in the chromosphere. They employed both spectroscopic and imaging data observed by AIA/SDO, HMI/SDO, FISS (Fast Imaging Solar Spectrograph), NIRIS (Near InfraRed Imaging Spectropolarimeter) installed at Big Bear Solar Observatory's $1.6 \mathrm{~m}$ New Solar Telescope (NST). However, there exist some previously quantitatively reported reconnection outflows e.g., fast reconnection outflow of $\sim 900-3500 \mathrm{~km} \mathrm{~s}^{-1}$ in a flare (Wang et al. 2007), redshifted outflow of $125 \mathrm{~km} \mathrm{~s}^{-1}$ (Tian et al. 2014), bidirectional outflows of $\sim 100 \mathrm{~km} \mathrm{~s}^{-1}$ in transition region explosive events (Innes et al. 1997). Hong et al. (2016) observed bidirectional outflows with a Doppler velocity of \pm (70-80) $\mathrm{km} \mathrm{s}^{-1}$ in the $\mathrm{H} \alpha$ line. Using magnetic field extrapolation, they estimated the position of the flux rope to be at a height of $\sim 900 \mathrm{~km}$ where the ambient magnetic field and mass density are to be $600 \mathrm{G}$ and $2.34 \times 10^{-10} \mathrm{~g} \mathrm{~cm}^{-3}$, respectively.

At the reconnection site, by taking into account the weak flare's F1 model, the local Alfvén speed has been measured to be $\sim 110 \mathrm{~km} \mathrm{~s}^{-1}$ (Machado et al. 1980). In the lower atmosphere, some small-scale activities e.g. Ellerman bombs (Archontis \& Hood 2009; Vissers et al. 2015) and hot explosions (Peter et al. 2014) have been reported as the consequences of magnetic reconnection. Hong et al. (2016) further emphasis on the similar mechanisms of the major and microflares even if there exists a huge difference in their energies.

Based on the observations of IRIS (Interface Region Imaging Spectrograph) and CHROMIS/SST (Swedish 1-m Solar Telescope), van der Voort et al. (2017) investigated the intermittent magnetic reconnection and its role in plasmoid formation during UV bursts occurring in the lower solar atmosphere. Their observations lead to a conclusion that the observed intermittent magnetic reconnection is being driven by plasmoid instability

Provornikova et al. (2016) studied the compression of plasma in the coronal magnetic reconnecting regions. The analytical considerations of first-order Fermi mechanism (de Gouveia dal Pino and Lazarian 2005) which can accelerate the particles bouncing between magnetized flows, converging in a reconnection region let them relate the spectral index of accelerated particles and total plasma compression. Their simulation suggested that sufficient plasma compression of factor 4 or higher can be achieved in the current sheets formed at magnetic nulls.

$\mathrm{Li}$ et al. (2016) analyzed an observational event of magnetic reconnection. This magnetic reconnection took place between an erupting filament and coronal loops in its vicinity. During the encounter of erupting filament with the coronal loops, an X-type structure was observed to form. They also observed current sheets and bidirectionally propagating plasmoids. They have also reported very clear evidence of successive magnetic reconnection which has been predicted by theory. Their observations also confirmed the theory of three-dimensional magnetic reconnection. They also estimated the mean magnetic energy release rate as well as the change of thermal energy during the time of reconnection which they found to be $(1.5 \pm 0.7) \times 10^{27} \mathrm{erg} \mathrm{s}^{-1}$ and $(4.0 \pm$ 3.1) $\times 10^{26} \mathrm{erg} \mathrm{s}^{-1}$.

Based on ground-based and spaceborne observations (AR 12597, 25 September 2016, 06:32 UT to 07:52 UT) of NVST (New Vacuum Solar Telescope), AIA (Atmospheric Imaging Assembly) and HMI (Helioseismic and Magnetic Imager), Huang et al. 2018 reported a magnetic reconnection event in the solar atmosphere. This event took place among AFS (Arch Filament System)'s threads and coronal loops. As a consequence of the relaxation of the twisted Arch Filament System, some of its threads encountered with the coronal loops further resulted in the inflows of reconnection.

Huang et al (2018) observed some interesting features e.g., remote brightening, fast flows and slow flows with multiple speeds. They found the origin of the fast flows in the reconnection 
region with an exceeding speed of approximately $1000 \mathrm{~km} \mathrm{~s}^{-1}$. However, they could not resolve if these flows are shock flows or just the sudden appearance of previously obscured coronal loops. The slow flows were estimated on the basis of three tracks of blobs and found to be 36,52 , and $81 \mathrm{~km} \mathrm{~s}^{-1}$. They interpreted the speed difference as the intermittent reconnection between multiple loop threads possessing different magnetic fields and plasma. As the remote brightening was observed away from the reconnection region but in the track of the fast flows. Hence, they interpreted these remote brightening as secondary activities being triggered by the fast flows.

It has been believed that CMEs demonstrate closed MFR (magnetic flux rope) like structures near the sun. On the other hand, when the CME propagates from the Sun to the interplanetary space, the magnitude of the magnetic field in the heliosphere continuously increases (Feng et al. 2018). The issue of the increased magnetic field can be resolved by considering the MFRs to be opened and disconnected through magnetic reconnection. Based on the observations of Wind spacecraft, Feng et al. (2018) have reported a magnetic cloud associated with a magnetic reconnection exhaust. Their observations revealed that magnetic reconnection caused disconnection of open field lines of magnetic cloud by joining the open field lines after the magnetic cloud.

By analyzing the imaging and spaceborne observations from Nançay Radioheliograph, AIA/SDO and HMI/SDO, Flanngain et al. (2018) studied a magnetic reconnection event occurred in a collapsing coronal loops system. During the partial collapse, the gradual and fast inflow phases were observed to be of the order of $1 \mathrm{~km} \mathrm{~s}^{-1}$ and $5 \mathrm{~km} \mathrm{~s}^{-1}$, respectively. However, they have recorded the fast and rapid outflows of the order of $30 \mathrm{~km} \mathrm{~s}^{-1}$ and 80 $100 \mathrm{~km} \mathrm{~s}^{-1}$, respectively. Interestingly, they estimated the reconnection rate to be $\sim 0.06$.

Based on the multiwavelength observations of AIA/SDO, Li et al. (2018) reported the coronal condensation during a magnetic reconnection event occurred among open and closed coronal loops. Magnetic reconnection plays an important role They reported the plasma cooling and condensation from $\sim 0.9 \mathrm{MK}$ to $\sim 0.6 \mathrm{MK}$, and then up to $\sim 0.05 \mathrm{MK}$. Their observations revealed that the condensation of hotter coronal plasma and its downflows is a consequence of magnetic reconnection. They further suggested that magnetic reconnection initiated the catastrophic cooling as well as condensation and played an important role in the mass cycle of coronal plasma. They observed the downflows of a mean speed of $60 \mathrm{~km} \mathrm{~s}^{-1}$ and also the condensation flowing first slowly and then rapidly with a speed of $100 \mathrm{~km} \mathrm{~s}^{-1}$.

Guglielmino et al. (2018), based on the IRIS observations of an emerging flux region associated with AR 12529, reported bidirectional high velocity flows of up to $100 \mathrm{~km} \mathrm{~s}^{-1}$. They suggested that these signatures may be an indication of multiple small scales, long-lived magnetic reconnection episodes occurred from the interaction of the ambient field with the emerging bipoles. However, they observed some flows along with the reconnected loops of $40-80 \mathrm{~km} \mathrm{~s}^{-1}$, interpreted as the consequence of intermittent reconnection.

Based on the AIA/SDO observations of an X-8.2 class flare dated 10 September 2017, Cheng et al. (2018) reported the formation of a super-hot current sheet and observed fragmented and turbulent magnetic reconnection inside it. The converging nature of bilateral plasmas towards the current sheet enabled them to detect the nonthermal motions and plasma heating. The plasma began to converge with an initial speed of $100 \mathrm{~km} \mathrm{~s}^{-1}$ which subsequently reduced to $\square 20 \mathrm{~km} \mathrm{~s}^{-1}$. This finding has been in good agreement with Liu et al. 2013, Zhu et al. 2016, Li et al. 2017, and Wang et al. 2017. The nonthermal velocities were estimated to be in a range of $\sim 100-150 \mathrm{~km} \mathrm{~s}^{-1}$. Such ha igh magnitude of non-thermal velocities indicates the existence of turbulent motions in the current sheet. They also found the intensity showing a power law behaviour with a spectral index of -
1.60 , very close to that of the turbulent current sheet. This fact leaded them to conclude that the current sheet has been fragmented in to different scaled structures. The intensity variations in other flaring regions following the power-law distribution and possessing the spectral indices in a range of -1.2 to -1.8 , strengthen the observations of a fragmented and turbulent reconnection.

Coronal loops connecting two active regions are called interconnected loops (ILs). If the two active regions are situated on opposite sides of the solar equator, they are said to be transequatorial loops (TLs). They were observed by Skylab in soft X-rays for the first time (SXRs; Chase et al. 1976). Based on the observations of XRT/Hinode and AIA/SDO, Du et al. (2018) reported the formation of interconnected loops which were formed at the northeastern limb. It is believed that the interconnected loops are formed as a consequence of magnetic reconnection in the higher solar corona and Du et al. (2018) concluded that the interconnected loops in their observations are formed by gradual magnetic reconnection. Interestingly, they observed that the interconnected loops were brightened in soft X-rays and EUVs which they found to occur because of the direct heating process of magnetic reconnection.

The phenomenon of mutual disappearance of two converging magnetic patches of opposite polarity seen in photospheric longitudinal magnetograms is called magnetic flux cancellation (Livi et al. 1985; Martin et al. 1985) and believed to be evidence of magnetic reconnection in the lower solar atmosphere (Priest et al. 1994). There exist two scenarios namely U-loop emergence and $\Omega$-loop submergence have been proposed for magnetic flux cancellation by Zwaan (1987). It has been found that the two magnetic patches which are unconnected can be connected via magnetic reconnection during the process of flux cancellation (Wang \& Shi 1993). The contribution of both the scenarios to the flux cancellation is highly dependent on the height at which the reconnection occurs. However, the U-loop emergence and $\Omega$-loop submergence scenarios will remain dominant when the magnetic reconnection will occur below and above the photosphere, respectively.

Employing spatial as well as temporal data provided by AIA, HMI, IRIS/SDO and XRT/ Hinode, Yang et al. (2018), studied the process of flux cancellation and observed multiple signatures of magnetic reconnection e.g., process of loop-loop interaction, formation of a sheet-like structure above the flux cancellation site and a chain of ejected plasma blobs.

Based on observations of an X 2.2 class flare in NOAA AR 12673 occurring on 06 September 2017, provided by AIA, HMI/SDO and GOES-13 satellites, Zou et al. (2019) reported a two-step magnetic reconnection. Initially, there was a magnetic flux rope before the flare whose one leg was rooted in a rotating sunspot and close to a null point. The sunspot motivated the magnetic flux rope to expand and as a result, the magnetic flux was pushed towards the null point. The null point reconnection created a disturbance which further triggered the second magnetic reconnection or the tether-cutting magnetic reconnection. Interestingly, the strapping flux of the rope was so strong that the rope could not be erupted by both the reconnections. By comparing this flare with an X 9.3 flare in the same region, they noticed a key difference between an eruptive and confined flare. They concluded that the magnetic flux rope is completely below and above the threshold of torus instability for confined and eruptive flares, respectively. They further concluded that magnetic reconnection independently may not trigger an eruption and a magnetohydrodynamic instability plays an important role in the eruption process.

Dynamical jets, a key for studying the decay of a Sunspot are found on the light bridges. So far, their formation mechanism is not fully understood. Data observed by Goode Solar Telescope, IRIS, Spectro-polarimeter/Hinode and AIA/SDO enabled Bai et al. (2019) to study the dynamical properties of fan-like light 
bridges. The $\mathrm{H} \alpha-0.8 \AA$ images revealed the propagation of ribbon-like brightenings along the light bridges which they considered as evidence of slipping reconnection. Based on their observations they suggested that the fan like jets are formed by magnetic reconnection and the photospheric convective motions have been playing a key in the triggering of magnetic reconnection.

\section{Conclusion}

Magnetic reconnection is a ubiquitous process occurring in astrophysical plasmas. This is the underlying process for most eruptive events taking place in astrophysical plasmas from the smallest (e.g., coronal bright points, jets) to large structures (e.g., flares, flux rope eruptions). This article has reviewed the SweetParker model, which dominated the reconnection world for decades, but was found to be not adequate to explain astrophysical eruptive events such as solar flares because of its slow magnetic reconnection rate. Petscheck's mechanism whose reconnection rate was found to be much faster became accepted as a suitable mechanism for explaining reconnection in solar eruptions. The present article also considered the developments made in the fastmagnetic reconnection after Petschek's mechanism.

Furthermore, the article focused on the observational events providing significant evidence of magnetic reconnection and sheds light on the advancements that have been made to understand magnetic reconnection through simulation and modelling. During the last decades, high-resolution numerical experiments such as those performed by Biskamp have been carried out to account for fast magnetic reconnection. These simulations have enriched our knowledge of magnetic reconnection and much emphasis has been given to plasmoid instability for explaining fast reconnection. Numerous numerical works have been carried out on the tearing instability in current sheets and formation of islands from the breakup of the current sheet. However, an exact theoretical explanation of the formation of plasmoids is still in its infancy. The article also sheds light on the observational progress that has been made for understanding the magnetic reconnection.

There exist numerous direct as well as indirect evidence of magnetic reconnection observed in the solar atmosphere but still, there are a few unanswered questions on the forefront of magnetic reconnection research. It has been accepted that the interconnected loop eruptions are important sources for coronal mass ejections and they are formed via magnetic reconnection occurring in the higher corona. However, the process of their formation and the release of associated energy in the solar corona is still not fully understood. There is a coexistence between solar flares and coronal eruptions but there exist even some X-class flares which occurred without an eruption. This is still not well understood how such flares took place and why they remain confined. It is also still not fully understood why the global reconnection rate is not always maximum and close to 0.1 ? Why the collisionless and MHD reconnection are distinctly different from anomalous resistivity and with a uniform resistivity? For reconnection in MHD, if the resistivity stands above a threshold with Lundquist number is $10^{4}$, the collisional reconnection follows the sweetparker outlines instead of attaining a rate of 0.1 . On the other hand, if the resistivity is constant but stands below the threshold, the reconnection rate attains a value of 0.01 with the sudden appearance of secondary islands. What is the reason that the Sweet-Parker reconnection does not happen at the maximum rate? When the secondary islands are generated and they accelerate the reconnection, why the reconnection rate remains limited to 0.01 and does not attain 0.1 .

In summary, even though our understanding about the physical processes governing magnetic reconnection has improved during the last years, there are still pending questions that need to be answered. With increasing high spatial and temporal resolution, detectors onboard spacecraft are helping to guide our understanding of magnetic reconnection in the solar corona.

\section{Acknowledgements:}

This work was supported by NNSFC grants 41731070 and 41574159, Key Research Program of Frontier Sciences-CAS, Grant No. QYZDJ-SSW-JSC028, Strategic Pioneer Program on Space Science, CAS, Grant No.XDA15052500 and in part by Research Fund from the Chinese Academy of Sciences and the Specialized Research Fund for State Key Laboratories of China. Authors are thankful to the referee for the careful analysis of the manuscript as well as valuable suggestions which have reasonably improved the quality of the manuscript.

\section{References:}

Archontis V. and Hood A. W., 2009, Astron. Astrophys. 508, 1469

Asai, A., Yokoyama, T., Shimojo, M., \& Shibata, K. 2004, ApJ, 605, L77

Aschwanden, M. J. 2004, Physics of the Solar Corona (Chichester: Praxis)

Bai, X., Navarro H. S., Siverio D. N. et al., 2019, Signatures of Magnetic Reconnection at the Footpoints of Fan-shaped Jets on a Light Bridge Driven by Photospheric Convective Motions, ApJ 870, 90

Balbus A., Hawley J. F., 1998, Rev. Mod. Phys. 70, 1

Baum P.J., Bratenahl A., 1974, Phys. Fluids 17, 1232

Bhattacharjee A., Huang Y.-M., Yang H., 2009, Fast reconnection in high-Lundquist-number plasmas due to the plasmoid Instability, PHYSICS OF PLASMAS 16, 112102

Bellan PM. 2000. Spheromaks. London: Imperial College Press

Birn, J. and Priest, E. R. (2007). Reconnection of Magnetic Fields: MHD and Collisionless Theory and Observations. (Cambridge University Press, Cambridge, UK).

Biskamp, D., Phys. Fluids 1986, Magnetic reconnection via current sheets, 29, 1520.

Biskamp, D., Magnetic Reconnection in Plasmas (Cambridge University Press, New York, 2000)

Bratenahl A. and Yeates C. M., 1970, Phys. Fluids 13, 2696

Carmichael, H., 1964 A process for flares, in Proceedings of AASNASA Symposium on the Physics of Solar Flares, ed. by W.N. Hess. NASA-SP 50, 451.

Cairns I. H., Lobzin V. V., Donea A. et al., 2018, Low Altitude Solar Magnetic Reconnection, Type III Solar Radio Bursts, and $X$-ray Emissions, Nature communications, 8

Cassak P. A., Liu Y. -H., Shay M. A., 2017, A review of the 0.1 reconnection rate problem, J. Plasma Phys. 83, 715830501

Chen P. F., and Shibata K., 2000, ApJ 545, 524

Chen, H., Zhang, J., Li, L., \& Ma, S. 2016, ApJL, 818, L27

Cheng X., Li Y., Wan L. F., 2018, Observations of Turbulent Magnetic Reconnection within a Solar Current Sheet, ApJ 866,64

de Gouveia dal Pino E. M. and Lazarian, A., 2005, Astron. Astrophys. 441, 845

Den Hartog D. J., Ahn J.-W., Almagri A. F. et al. 2007, Nucl. Fusion 47,17

Dungey, J. W., 1953, Conditions for the occurrence of electrical discharges in astrophysical systems, The London, Edinburgh, and Dublin Philosophical Magazine and Journal of Science, 44:354,725

Du G., Chen Y., Zhu C., 2018, Formation of Large-scale Coronal Loops Interconnecting Two Active Regions through Gradual Magnetic Reconnection and an Associated Heating Process, ApJ 860,40

Egedal J., Fasoli A., Porkolab M. et al., 2000, Rev. Sci. Instrum. 71,3351

Egedal J., Fasoli A., Nazemi J., 2003, Phys. Rev. Lett. 90, 135003

Flannagain A. M. O., Maloney S. A., Gallagher P. T., 2018, Threedimensional magnetic reconnection in a collapsing coronal loop system, Astron. Astrophys. 617, A9.

Feng H. Q., Wang J. M., Zhao G. Q., 2018, Observations on the Magnetic Disconnections of a Magnetic Cloud from the Sun through Magnetic Reconnection, ApJ 864, 101

Fiksel G., Fox W., Bhattacharjee A. et al. 2014, Magnetic Reconnection between Colliding Magnetized Laser-Produced Plasma Plumes, PRL 113, 105003

Fox W., Bhattacharjee A., and Germaschewski K., 2011, Fast Magnetic Reconnection in Laser-Produced Plasma Bubbles, Phy. Rev. Lett. 106, 215003 
Gonzalez, W., Parker E., 2016, Magnetic Reconnection: Concepts and applications, Springer.

Guglielmino S. L., Zuccarello F., Young P. R., 2018, IRIS Observations of Magnetic Interactions in the Solar Atmosphere between Preexisting and Emerging Magnetic Fields. I. Overall Evolution, ApJ 856, 127

Huang Y.-M., Bhattacharjee A., 2010, Scaling laws of resistive magnetohydrodynamic reconnection in the high-Lundquistnumber, plasmoid-unstable regime, Physics of Plasmas 17, 062104

Huang Y.-M., Bhattacharjee A., 2013, Plasmoid instability in highLundquist-number magnetic reconnection, Physics of Plasmas 20, 055702

Huang Z., Mou C., Fu H., 2018, A Magnetic Reconnection Event in the Solar Atmosphere Driven by Relaxation of a Twisted Arch Filament System, ApJ 853, L26

Innes, D. E., Guo, L.-J., Huang, Y.-M., Bhattacharjee, A. 2015, IRIS Si IV line profiles: An indication for the plasmoid instability during small-scale magnetic reconnection on the Sun, ApJ, 813, 86.

Innes D. E., Inhester B., Axford W. I., and Wilhelm K., 1997, Natur 386,811

Yamada, Ji H., M., Hsu, S., Kulsrud, R. 1998, Experimental test of the Sweet-Parker model of magnetic reconnection. Phys. Rev. Lett. 80, 3256.

Ji H., Almagri A., Prager S., and Sar J., 1994. Phys. Rev. Lett. 73, 668

Jiang Y., Bi Y., Yang J, et al., 2013, ApJ 775, 132

Joglekar A. S., Thomas A. G. R., Fox W. et al. 2014, Magnetic Reconnection in Plasma under Inertial Confinement Fusion Conditions Driven by Heat Flux Effects in Ohm's Law, Phy. Rev. Lett. 112, 105004

Goff, C. P., van Driel-Gesztelyi, L., Harra, L. K., et al. 2005 , Direct observation of high-speed plasma outflows produced by magnetic reconnection in solar impulsive events, Astron. Astrophys. 434, 761

Hong J., Ding M. D., Li Y., 2016, Bidirectional outflows as evidence of magnetic reconnection leading to a solar microflare, ApJ 820, L 17

Kivelson, M.G. and Russell, C.T. 1995, Introduction to Space Physics, Cambridge University Press, Cambridge

Knox S. O., Barnes C. W., Marklin G. J. et al. 1986, Phys. Rev. Lett. 56, 842

Kadomtsev B., 1975, Sov. J. Plasma Phys. 1, 389

Lin, J., Ko, Y.-K., Sui, L., Raymond, J. C., Stenborg, G. A., Jiang, Y., Zhao, S., Mancuso, S. 2005, ApJ, 622, 1251

Lin J., and Forbes T. G., 2000, JGR 105, 2375

Li, L. and Zhang, J., 2009, Observations of the magnetic reconnection signature of an M2 flare on 2000 March 23. ApJ, 703, 877.

Li L., Zhang J., Peter H. et al., 2016, Magnetic reconnection between a solar filament and nearby coronal loops, Nature Physics 12, 847

Li, Y., Xue, J. C., Ding, M. D. et al. 2018, Spectroscopic Observations of a Current Sheet in a Solar Flare, ApJL, 853, L15

Li C. K., Séguin F. H., Frenje J. A. et al. 2007, Observation of Megagauss-Field Topology Changes due to Magnetic Reconnection in Laser-Produced Plasmas, Phy. Rev. Lett. PRL 99, 055001

Li, L. P., Peter, H., Chen, F., \& Zhang, J. 2014, A\&A, 570, A93

Li, L. P., and Zhang, J., 2009, ApJ, 703, 877

Li L., Zhang J., Peter H., 2018 Coronal Condensations Caused by Magnetic Reconnection between Solar Coronal Loops, ApJ 864 L4.

Li, Y., Sun, X., Ding, M. D., Qiu, J., \& Priest, E. R. 2017, ApJ, 835, 190

Lin, J., Ko, Y.-K., Sui, L., et al. 2005, ApJ, 622, 1251

Liu, R., Lee, J., Wang, T., et al. 2010, ApJL, 723, L28

Liu, W., Chen, Q., \& Petrosian, V. 2013, ApJ, 767, 168

Liu, W., Chen, Q., \& Petrosian, V. 2013, ApJ, 767, 168 Livi, S. H. B., Wang, J., \& Martin, S. F. 1985, AuJPh, 38, 855 Martin, S. F., Livi, S. H. B., \& Wang, J. 1985, AuJPh, 38, 929

Liu, R., Lee, J., Wang, T., et al. 2010, ApJ, 723, L28

Loureiro N. F., Samtaney R., Schekochihin A. A., et al. 2012, Magnetic reconnection and stochastic plasmoid chains in
high-Lundquist-number plasmas, Physics of Plasmas 19, 042303

Marsch E., 2006, Kinetic Physics of the Solar Corona and Solar Wind, Living Rev. Solar Phys., 3, 1

Meyer-Vernet, N. (2007). Basics of the Solar Wind. (Cambridge University Press, Cambridge, UK).

Masuda, S., Kosugi, T., H. Hara, S. Tsuneta, Y. Ogawara, 1994, A loop-top hard X-ray source in a compact solar flare as evidence for magnetic reconnection. Nature 371, 495.

McKenzie, D. E., \& Hudson, H. S. 1999, X-Ray Observations of Motions and Structure above a Solar Flare Arcade, ApJ, 519, 193

Mei Z. X., Keppens R., Roussev I. I., Lin J., 2017, Magnetic reconnection during eruptive magnetic flux ropes, Astron. Astrophys. 604, L7

Nagayama Y., McGuire K. M., Bitter M., Cavallo A., Fredrickson E. D., et al. 1991. Phys. Rev. Lett. 67, 3527

Nagayama Y., Yamada M., Park W., Fredrickson E. D., Janos A. C., et al. 1996, Phys. Plasmas 3, 1647

Nilson P. M., Willingale L., Kaluza M. C., et al. 2006, Magnetic Reconnection and Plasma Dynamics in Two-Beam Laser-Solid Interactions, Phy. Rev. Lett. 97, 255001

Ning, Z. and Guo, Y., 2014, Investigation of the moving structures in a coronal bright point, ApJ, 794:79

Ono, Y, Yamada, M., Tajima, T. \& Matsumoto, R. (1996). Ion acceleration and direct ion heating in three-component magnetic reconnection, Phys. Rev.Lett, 76, 3328

Nishizuka, N., Takasaki, H., Asai, A., \& Shibata, K. 2010, ApJ, 711, 1062

Ono Y., Ellis R. A. Jr, Janos A. C., Levinton F. M. et al. 1988, Phys. Rev. Lett. 61, 2847

Ono Y., Morita A., Katsurai M., et al. 1993, Phys. Plasmas 4, 1953

Parker, E.N. 1963a The Solar-Flare Phenomenon and the Theory of Reconnection and Annihilation of Magnetic Fields, ApJS 8, 177

Parker, E.N. 1972, Topological Dissipation and the Small-Scale Fields in Turbulent GasesApJ 174, 499.

Parker, E.N. 1979, Cosmical Magnetic Fields

Parker, E.N. 1983, Magnetic Neutral Sheets in Evolving Fields Part Two - Formation of the Solar CoronaApJ 264, 642

Parker, E. N. (1963b). The solar flare phenomenon and the theory of reconnection and annihilation of magnetic fields. Astrophys. J. 8, 177.

Petschek, H. E.: 1964, in W. N. Hess (ed.), Symp. on the Physics of Solar Flares, National Aero. And Space Adm., Washington, NASA SP 50, p. 425

Peter H., Tian H., Curdt W., et al. 2014, Sci 346, 1255726

Pontin D. I., 2011, Three-dimensional magnetic reconnection regimes: Advances in Space Res. 47, 1508

Priest, E. R. 1982, Solar Magnetohydrodynamics (Boston: Reidel), 127

Priest, E., \& T. Forbes, 2000, Magnetic Reconnection: MHD Theory and Applications, Cambridge University Press, Cambridge

Priest E. R., Parnell C. E. and Martin S. F., 1994, ApJ 427, 459

Provornikova E., Laming J. M., and Lukin V. S., 2016, plasma compression in magnetic reconnection regions in the solar corona, ApJ 825, 55

Qiu, J., Wang H., Cheng, C. Z., 2004, Magnetic reconnection and mass acceleration in flare-coronal mass ejection events, 2004, ApJ 604, 900

Ren Y., Yamada M., Gerhardt S. et al. 2005, Phy. Rev. Lett. 95, 055003

Rosenberg M. J., Li C. K., Fox W. et al. 2015, Slowing of Magnetic Reconnection Concurrent with Weakening Plasma Inflows and Increasing Collisionality in Strongly Driven Laser-Plasma Experiments, Phy. Rev. Lett. 114, 205004

Rosenberg M. J., Li C. K., Fox W. et al. 2014, A laboratory study of asymmetric magnetic reconnection in strongly driven plasmas, Nature Communications 6, 6190

Sakai, J.-I., \& de Jager, C. 1996, SSRv, 77, 1

Sarff J., Almagri A., Anderson J., Brower D., Craig D., et al. 2005. In the magnetized plasma in galaxy evolution, ed. Chyzy K. T., Otmianowska-Mazur K., Soida M., Dettmar R. -J., pp. 4855

Savage, S. L., McKenzie, D. E. 2011, Quantitative Examination of a Large Sample of Supra-arcade Downflows in Eruptive Solar Flares ApJ, 730, 98 
Savage, S. L., McKenzie, D. E., Reeves, K. K., Forbes, T. G., and Longcope, D. W. 2010, ApJ, 722, 329

Shen Y., Liu Y., and Su J., 2012, ApJ 750, 12

Shibata, K., Masuda, S., Shimojo, M., Hara, H., Yokoyama, T. Tsuneta, S., Kosugi, T., and Ogawara, Y., 1995, Astrophys. J. (Letters) 451, L83

Shibata K., Yokoyama T., Shimojo M. 1996b, Advances in Space Res. 17, 197

Shibata K., 1996a, Advances in Space Res. 17, 9

Shibata, K., Masuda, S., Shimojo, M., et al. 1995, ApJ, 451, L83

Somov, B.V. 2000, Cosmic Plasma Physics, Astrophysics and Space Science Library, Vol. 251, Kluwer Academic Publishers, Dordrecht, The Netherlands.

Stenzel, R.L. \& Gekelman, W. (1981). Magnetic field line reconnection experiments. 1. Field topologies, J. Geophys. Res. 86, 649-58.

Stenzel R., Gekelman W., 1979, Geophys. Phys. Res. 86, 649

Sturrock, P. A. 1966, Model of the high-energy phase of solar flares. Nature 211, 695

Sturrock, P.A. \& Uchida, Y. 1981, Coronal heating by stochastic magnetic pumping, ApJ 246, 331.

Syrovatskii S.I., 1981, Annu. Rev. Astron. Astrophys. 19, 163

Van Ballegooijen, A.A. 1986, Cascade of Magnetic Energy as a Mechanism of Coronal Heating, ApJ 311, 1001.

Sturrock, P. A. 1994 Plasma Physics - An Introduction to the Theory of Astrophysical, Geophysical and Laboratory Plasmas (Cambridge: Cambridge University Press).

Sui, L. and Holman, G. D. 2003, ApJ, 596, L251

Su Y., Veronig A. M., Holman G. D. et al., 2013, Imaging corona magnetic-field reconnection in a solar flare, Nature Phys. 9, 489

Sun J Q., Cheng X., Ding M. D., 2015, Extreme ultraviolet imaging of three-dimensional magnetic reconnection in a solar eruption, Nature Communications 6, 7598

Sweet, P. A. (1958b). The production of high energy particles in solar flares. Nuovo Cimento 8,188-196

Tsinganos, K., Sauty, C., Surlantzis, G., Trussoni, E., and Contopoulos, J. 1996, On the relation of limiting characteristics to critical surfaces in magnetohydrodynamic flows, MNRAS, 283, 811.

E.R. Priest, T.G. Forbes, 1986, New models for fast steady-state magnetic reconnection. J. Geophys. Res. 91(A5), 5579.

Sweet, P.A. (1958) The production of high energy particles in solar flares, Nuovo Cimento Suppl. 8, Ser. X, 188

Sweet, P.A. in Electromagnetic Phenomena in Cosmical Physics, ed. by B. Lehnert. IAU Symposium, vol. 6 (1958), p. 123

Tajima, T. \& Shibata, K. 2002, Plasma Astrophysics, Perseus Publishing, Cambridge, Massachusetts

Takasao, S., Asai, A., Isobe, H., Shibata, K. 2012, Simultaneous Observation of Reconnection Inflow and Outflow Associated with the 2010 August 18 Solar Flare, ApJ, 745, L6

Tian H., Li G., Reeves K. K., et al. 2014, ApJ 797, L14

Treumann, R.A. and Baumjohann, W. 1997, Advanced Space Plasma Physics, Imperial College Press, London

Tsuneta, S. et al, Observation of a solar flare at the limb with the YOHKOH soft X-ray telescope. Publ. Astron. Soc. Jpn. 44, L63-L69 (1992)

Tsuneta S. 1996. Structure and Dynamics of Magnetic Reconnection in a Solar Flare, ApJ. 456:840

Tsuneta, S., Masuda, S., Kosugi, T., and Sato, J. (1997). Hot and superhot plasmas above an impulsive flare loop. ApJ 478 , 787-798.

van der Voort L. R., De Pontieu B., Scharmer, G. B., et al. 2017, Intermittent Reconnection and Plasmoids in UV Bursts in the Low Solar Atmosphere, ApJ $851 \mathrm{~L} 6$

Vissers G. J. M., Rouppe van der Voort L. H. M., Rutten, R. J. et al. 2015, ApJ 812, 11

Wang, T. J., Sui, L., and Jiong Qiu, J. 2007, Direct observation of high-speed plasma outflows produced by magnetic reconnection in solar impulsive events, ApJ 661, L207.

Wang, J., \& Shi, Z. 1993, Solar Phys, 143, 119

Wang, J., Simões, P. J. A., Jeffrey, N. L. S., et al. 2017, ApJ, 847, L1

Weller, H. R., and Roberson, N.M., 1980, Capture reactions with protons, neutrons, and alpha particles, Rev. Mod. Phys. 52, 699

Wesson J., 1987, Tokamaks. London, Clarendon Press
Webb, D. F., Burkepile, J., Forbes, T. G., \& Riley, P. 2003, JGR, 108, 1440 Wheatland, M. S., Sturrock, P. A., \& Roumeliotis, G. 2000, ApJ, 540, 1150 Wiegelmann, T. 2004, SoPh, 219, 87

Xue Z., Yan X., Cheng X. et al., 2016, Observing the release of a twist by magnetic reconnection in a solar filament eruption, Nature Communication 7,11837

Yan, X. L., Yang, L. H., Xue, Z. K., et al. 2018, ApJL, 853, L18

Yang, S., Zhang, J., Xiang, Y. 2015, ApJ, 798, L11

Yang, B., Yang, J., Bi, Y. et al. 2018, Observational Evidence of Magnetic Reconnection Associated with Magnetic Flux Cancellation, ApJ, 861, 135

Yamada, M., Kulsrud, R., Ji, H. 2010, Magnetic Reconnection. Rev. Mod. Phys. 82, 603.

E.N. Parker, J. 1957, Sweet's Mechanism for Merging Magnetic Fields in Conducting FluidGeophys. Res. 62, 509.

Yamada, M., Ono, Y., Hayakawa, A., and Katsurai, M. 1990, Magnetic reconnection of plasma toroids with cohelicity and counterhelicity. Phys. Rev. Lett. 65, 721.

Yamada M, Ji H, Hsu S, Carter T, Kulsrud R, et al. 1997b. Phys. Rev. Lett. 78:3117

Yamada M., Levinton F., Pomphrey N., Budny R., Manickam J., Nagayama Y., 1994, Phys. Plasmas 1, 3269

Yamada M., Ji H., Hsu S. et al., 1997a, Phys. Plasmas 4, 1936

Yamada M., Ji H., Hsu S. et al. 1997b, Phys. Rev. Lett. 78, 3117

Yamada M., Ono Y., Hayakawa A., et al. 1990, Phys. Rev. Lett. 65, 721

Yamada M., Kulsrud R., and Ji H., 2010, Magnetic reconnection, Rev. Modern Phys. 82, 603

Yokoyama, T., Akita, K., Morimoto, T., Inoue, K., and Newmark, J. (2001). Clear evidence of reconnection inflow of a solar flare. ApJ546, L69-L72.

Yang B., Yang J., Bi Y. et al. 2018, Observational Evidence of Magnetic Reconnection Associated with Magnetic Flux Cancellation, ApJ 861, 135.

Yang, S., Zhang, J., Xiang, Y., 2015, Magnetic reconnection between small-scale loops observed with the new vacuum solar telescope, $A p J, 98, \mathrm{~L} 11$

Zhang, Q. M. Chen, P. F., Guo, Y., et al. 2012, Two types of magnetic reconnection in coronal bright points and the corresponding magnetic configuration, $A p J, 746,19$.

Zhong J., Li Y., Wang X., 2010, Modelling loop-top X-ray source and reconnection outflows in solar flares with intense lasers, Nature Phys. 6, 984

Zhou G. P., Zhang J., Wang J. X., and Wheatland M. S., 2017, ApJ $851, \mathrm{~L} 1$

Zhu, C., Liu, R., Alexander, D., \& McAteer, R. T. J. 2016, ApJ, 821, L29

Zou P., Jiang C., Feng X. et al. 2019, A Two-step Magnetic Reconnection in a Confined X-class Flare in Solar Active Region 12673, ApJ 870, 97

Zwaan, C., 1987, ARA\&A, 25, 83

Zweibel, E. G. and Yamada, M. 2009, Perspectives on magnetic reconnection, Proc. R. Soc. A 472, 20160479

Zweibel, E. G. and Yamada, M. 2009, Magnetic Reconnection in Astrophysical and Laboratory Plasmas, Annu. Rev. Astron. Astrophys. 2009, 47, 291 